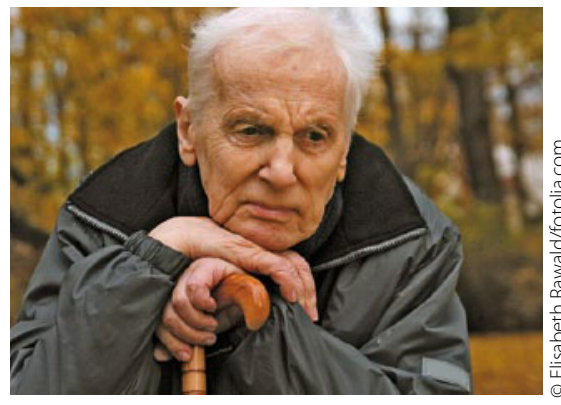

Er klagt nur über Schmerzen - fragen Sie auch nach einer Depression!

- Zwei Drittel der depressiven Personen suchen wegen körperlich-schmerzhafter Beschwerden ihren Hausarzt auf. Daneben geben sie meist unspezifische Beschwerden wie Schlafstörungen, Appetitminderung und Kraftlosigkeit an. Hier sollte der Hausarzt nach den Hauptsymptomen einer Depression - gedrückte Stimmung, Interesse- und Freudlosigkeit sowie Antriebstörungen - aktiv fragen. Der selektive Serotonin- und Noradrenalin-Wiederaufnahmehemmer (SSNRI) Duloxetin (Cymbalta ${ }^{\circledR}$ ) kann bei diesen Patienten eine effektive

Wenn die Seele schmerzt

\title{
Welches Antidepressivum ist geeignet?
}

Therapieoption darstellen. Wie die Beobachtungsstudie PADRE an über $4500 \mathrm{~Pa}$ tienten mit akuter depressiver Episode ergab, hatten nach Einschätzung der Ärzte $87,8 \%$ relevante Schmerzsymptome [Schneider E et al. Poster DGSS 2009, Berlin]. Als Erklärung wird eine Dysregulation von Serotonin und Noradrenalin angenommen, die bei der Depression eine entscheidende Bedeutung hat, sagte Dr. Michael Lorrain, Düsseldorf. Aufgrund dieser Imbalance haben die Patienten möglicherweise ein verändertes Schmerzempfinden.

Die Wirksamkeit von Duloxetin wurde in einer Analyse von gepoolten Daten zweier randomisierter, doppelblinder und placebokontrollierter Studien bestätigt [Fava M et al. J Clin Psychiatry 2004; 65: 521-530]. Darin führte die Therapie mit Duloxetin über neun Wochen zu einer signifikanten Verbesserung der gesamten
Schmerzen sowie der Rücken- und Schulterschmerzen gegenüber Placebo [Detke MJ et al. J Clin Psychiatry 2002; 63: 308315].

Aufgrund der Datenlage empfahl Lorrain, Duloxetin insbesondere bei körperlichen Beschwerden im Rahmen einer Depression einzusetzen. Den Erfahrungen des Psychiaters zufolge könne eine Startdosis von täglich $30 \mathrm{mg}$ über eine Woche das Auftreten von Übelkeit signifikant reduzieren. Es gelte zudem, energisch und unterstützend zuzuwarten. Die übliche Erhaltungsdosis von Duloxetin beträgt 60 $\mathrm{mg} /$ Tag. Eine Dosissteigerung auf bis zu $120 \mathrm{mg}$ täglich ist möglich und sollte bei Bedarf auch ausgeschöpft werden.

- Dr. Ralph Hausmann

Quelle: Pressegespräch:„Der depressive Patient in der Praxis - eine Mission für Zwei", Kaiserslautern, Mai 2011 (Veranstalter: Lilly)

\section{Patienten mit KHK, Hypercholesterinämie oder nach Schlaganfall Hoch dosiertes Atorvastatin verbessert ihre Chancen}

— Noch vor wenigen Jahren waren Experten der Meinung, dass eine moderate Absenkung von LDL-Cholesterin zur Prävention kardiovaskulärer Ereignisse ausreicht. Heute ist klar, dass der Nutzen einer lipidsenkenden Behandlung nicht bei einem LDLCholesterin von 100 mg/dl endet. In nationalen und internationalen Richtlinien wird ein Zielwert von $70 \mathrm{mg} / \mathrm{dl}$ bei erhöhtem kardiovaskulären Risiko empfohlen.

Der entscheidende Beleg gelang in der TNT-Studie [LaRosa JC et al. N Engl J Med. 2005; 352:1425-1435], in der Atorvastatin (Sortis ${ }^{\oplus}$ ) $10 \mathrm{mg}$ und $80 \mathrm{mg}$ miteinander verglichen wurden. 10001 Patienten mit manifester, stabiler KHK und Hypercholesterinämie wurden im Median 4,9 Jahre behandelt. Unter $10 \mathrm{mg}$ Atorvastatin traten insgesamt 548 primäre Endpunkte (tödliche KHK, nicht tödlicher Herzinfarkt, Wiederbelebung nach Herzstillstand, tödlicher und nicht tödlicher Schlaganfall) auf, unter $80 \mathrm{mg}$ Atorvastatin nur 434 Ereignisse. Die- ser signifikante Unterschied von 22\% war überwiegend durch die Reduktion der Herzinfarktrate und der Schlaganfallhäufigkeit bestimmt.

\section{Auch wirksam in der Schlaganfall-Sekundärprävention}

Darüber hinaus wurden für eine Hochdosisbehandlung mit Atorvastatin neue Therapiefelder erschlossen. Die SPARCL-Studie [SPARCL Investigators. N Engl J Med. 2006; 355: 549-559] ist die bisher einzige Endpunktstudie, in der die Wirkung eines Statins in der Sekundärprävention des Schlaganfalls untersucht wurde. Dazu wurden 4731 Patienten nach Schlaganfall oder TIA ohne manifeste KHK randomisiert entweder mit $80 \mathrm{mg}$ Atorvastatin oder Placebo über median 4,9 Jahre behandelt.

Der primäre Endpunkt (erneutes Auftreten eines tödlichen oder nicht tödlichen Schlaganfalls) wurde unter Atorvastatin im Vergleich zu Placebo signifikant um 16\% reduziert. Diese Ergebnisse sind inzwischen fester Bestandteil der SchlaganfallLeitlinien der Deutschen Gesellschaft für Neurologie zur Behandlung und Prävention eines Schlaganfalls [Diener $\mathrm{HC}$ et al. Akt Neurol. 2007; 34: 8-12].

In einer Metaanalyse aus 44 abgeschlossenen Studien [Newman CB et al. Am J Cardiol. 2003; 92: 670-676] mit insgesamt über 16000 Patienten (9000 mit Atorvastatin, etwa 1800 mit Placebo und etwa 5300 mit anderen Statinen behandelt) erwies sich Atorvastatin über den gesamten Dosisbereich von 10-80 mg als gut verträglich. Auch für die höchste Dosis von $80 \mathrm{mg}$ war die Häufigkeit substanzbedingter Nebenwirkungen - einschließlich muskulärer - nicht erhöht und mit Placebo vergleichbar. Es wurde von keinen substanzbedingten Rhabdomyolysen berichtet.

- Red.

Quelle: Nach Informationen von Pfizer 